\title{
FÍSTULA ARTERIOVENOSA EM CRIANÇAS E ADULTOS JOVENS
}

\section{ARTERIOVENOUS FISTULA IN CHILDREN AND YOUNG ADULTS}

\author{
Marcio Lopes Miranda ${ }^{1}$ \\ Marcia Alessandra Cavalaro ${ }^{2}$ \\ Antonio Gonçalves Oliveira Filho, TCBC-SE ${ }^{1}$ \\ Liliane Cury Prates ${ }^{3}$ \\ Vera Maria Santoro Belangero ${ }^{4}$ \\ Joaquim Murray Bustorff-Silva ${ }^{5}$
}

\begin{abstract}
RESUMO: Objetivo: Com a introdução e o aprimoramento de técnicas para a realização de fístulas arteriovenosas (FAV) em crianças, pacientes renais crônicos puderam iniciar tratamento com hemodiálise sem a necessidade de uso de shunts externos. Métodos: Com o objetivo avaliar os resultados da confecção de fístulas arteriovenosas em crianças e adultos jovens de baixo peso e os fatores de risco relacionados. Foram levantados retrospectivamente os prontuários de 31 pacientes submetidos a $35 \mathrm{FAV}$, com idade variando de cinco a 24 anos, com peso entre 16 e 50kg. Os fatores de risco estudados foram hipertensão arterial, antecedentes de trombose, tendência à hipovolemia e presença de hipercolesterolemia. Resultados: O índice de perda observado foi de $25 \%$ em pacientes com menos de $20 \mathrm{~kg}$ e de $22,2 \%$ naqueles com mais de $20 \mathrm{~kg}$, resultados comparáveis aos da literatura. Conclusões: Observou-se que fístulas proximais não sofreram trombose; não houve relação entre a perda da FAV e os fatores de risco estudados e observou-se um índice de perda tardia de $23,5 \%$ nos pacientes que não estavam sendo submetidos à hemodiálise. Nos pacientes que utilizavam a FAV não se observou perda da mesma.
\end{abstract}

Descritores: Fístula arteriovenosa; Hemodiálise; Pediatria; Microcirurgia.

\section{INTRODUÇÃO}

Antes de 1960, poucas crianças renais crônicas eram tratadas com hemodiálise. Com o advento do shunt arteriovenoso por Quinton-Scribner ${ }^{1}$ em 1960, o tratamento hemodialítico de longa duração tornou-se uma opção terapêutica viável. Em 1966 Brescia e Cimino ${ }^{2}$ descreveram uma nova técnica para confecção de fístula arteriovenosa (FAV) em adultos em hemodiálise crônica, diminuindo-se, assim, a necessidade de shunts externos e evitando-se suas complicações, como infecção, isquemia e trombose. Da mesma forma, foram aprimoradas as técnicas de confecção de FAV em crianças. Isso permitiu o gradual abandono dos shunts que, quando implantados em crianças, se tornavam ainda menos adequados, fazendo-as sentirem-se restritas em suas atividades físicas e trazendo também desordens psicológi- cas, já que a presença de um cateter externo sempre as fazia lembrar de sua doença ${ }^{3}$.

Apesar das FAV terem se mostrado uma boa via para hemodiálise em adultos, vários autores descrevem dificuldades em obter o mesmo sucesso em crianças pequenas, devido às limitações impostas pelo pequeno calibre dos vasos e ao consequiente baixo fluxo através da anastomose ${ }^{3,4}$. Em 1983, Kinnaert el al. ${ }^{5}$ utilizaram a técnica descrita por Gracz et al. ${ }^{6}$ para confeccionar FAV no nível do cotovelo em crianças de até $23 \mathrm{~kg}$, e em 1981 Bourquelot et al. ${ }^{7}$ descreveram a primeira FAV distal em crianças pesando menos de $10 \mathrm{~kg}$, utilizando técnicas microcirúrgicas. Desde então, outros autores têm relatado maior taxa de sucesso nesse tipo de procedimento ${ }^{8-13}$.

O objetivo deste trabalho foi analisar a evolução de crianças e adultos jovens com insuficiência renal crônica submetidos à confecção de fístula arteriovenosa pela Dis-

1. Professor Assistente da Disciplina de Cirurgia Pediátrica do HC/UNICAMP

2. Residente da Disciplina de Cirurgia Pediátrica do HC/UNICAMP

3. Professora Assistente do Serviço de Nefropediatria do HC/UNICAMP

4. Chefe do Serviço de Nefropediatria do HC/UNICAMP

5. Chefe da Disciplina de Cirurgia Pediátrica do HC/UNICAMP

Recebido em 28/3/2000

Aceito para publicação em 25/8/2000

Trabalho realizado no Hospital de Clínicas da Faculdade de Ciências Médicas da Universidade Estadual de Campinas UNICAMP 
ciplina de Cirurgia Pediátrica, avaliando-se a técnica cirúrgica utilizada, a sua localização e os fatores relacionados com suas complicações.

\section{MÉ TODO}

Entre janeiro de 1996 e abril de 1999, 31 pacientes foram submetidos à confecção de 35 fístulas arteriovenosas (FAV) para acesso para hemodiálise crônica. Em relação ao sexo dos pacientes, 17 eram meninas e 14 eram meninos. A idade média foi de 13 anos (mediana=13,1) (Gráfico 1), variando de cinco a 24 anos e o peso médio= $32 \mathrm{~kg}$ (16 a 50kg). A insuficiência renal crônica foi causada por glomerulopatia em 14 casos e por alterações do trato geniturinário (uropatia) em 17 casos. A média dos níveis séricos de creatinina foi de $7,2 \pm 3,5 \mathrm{mg} / \mathrm{dl}$, de uréia $117 \pm 41,4 \mathrm{mg} / \mathrm{dl}$ e de hemoglobina de $9,2 \pm 2,2 \mathrm{mg} \%$.

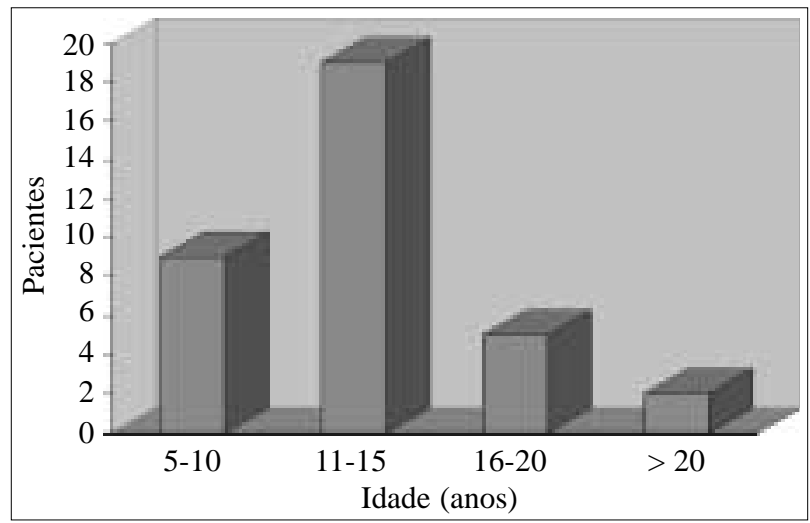

Gráfico 1 - Distribuição dos pacientes de acordo com a idade.

A indicação para a confecção da FAV foi feita de acordo com Protocolo da Disciplina de Nefropediatria, sendo dependente do estágio da insuficiência renal. Pacientes com clearance de creatinina entre 10 e $50 \mathrm{ml} / \mathrm{min} / 1,73 \mathrm{~m} 2$ de superfície corporal são tratados conservadoramente (vitamina $\mathrm{D}$, complexo vitamínico $\mathrm{B}$, ácido fólico, eritropoetina) e pacientes com clearance de creatinina $<10 \mathrm{ml} / \mathrm{min} /$ $1,73 \mathrm{~m} 2$ entram em esquema de diálise peritoneal ou hemodiálise. A confecção da FAV foi programada em pacientes com níveis decrescentes de clearance de creatinina (entre 10 e $20 \mathrm{ml} / \mathrm{min} / 1,73 \mathrm{~m} 2$ ).

Os pacientes foram avaliados no peri-operatório com relação ao valor de níveis pressóricos, fatores favorecedores para trombose (antecedente de trombose de rim transplantado, lúpus eritematoso sistêmico, plaquetose, hematócrito elevado p.ex.), tendência à hipovolemia (pacientes poliúricos), presença de hipercolesterolemia, tempo e tipo de diálise peritoneal prévia.

A avaliação pré-operatória consistiu em exame físico e visualização dos vasos após garroteamento do membro e obtenção dos níveis séricos de sódio, potássio, uréia, creatinina e hemoglobina. O procedimento foi realizado preferencialmente no membro não dominante, com o paciente sob anestesia geral associada a bloqueio do plexo bra- quial. Foram utilizadas lentes de magnificação (4x) para realização do procedimento, tendo sido os vasos clampeados e heparinizados durante o tempo de anastomose, que foi término-lateral, com sutura contínua com fio monofilamentar de polipropileno 7-0 (Figura 1). A escolha do local para confecção da FAV dependeu da observação venosa superficial e da existência de cirurgia anterior. As fístulas distais foram confeccionadas entre a veia cefálica e a artéria radial, e as proximais entre a veia cefálica ou basílica e a artéria braquial. A viabilidade das FAV foi avaliada através do exame físico (palpação de frêmito/pulso) e a facilidade para punção da mesma.

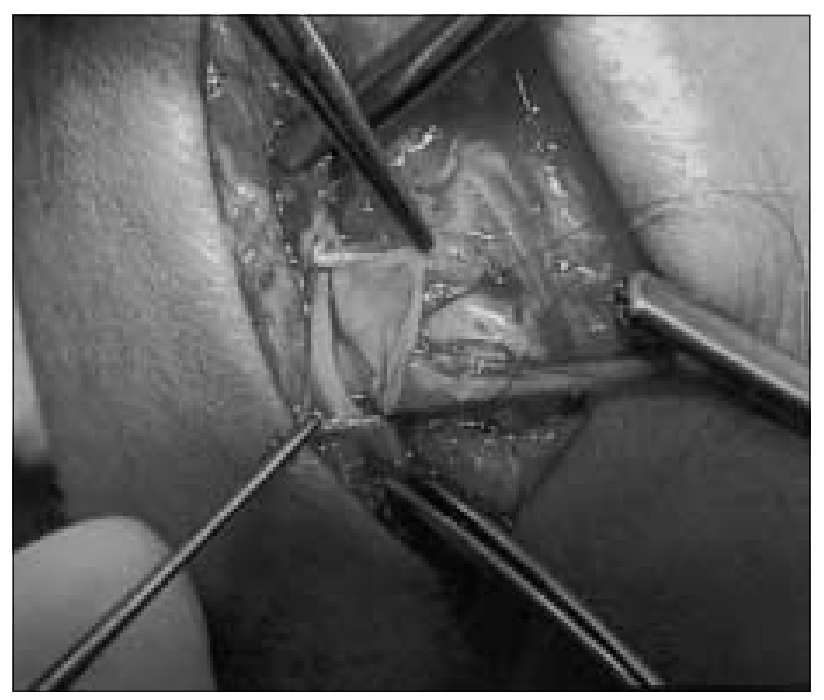

Figura 1 - Veia e artéria pinçadas, sendo anastomosadas término-lateralmente com fio monofilamentar de polipropileno 7-0.

A diálise peritoneal e a medicação anti-hipertensiva habitual foram suspensas nas primeiras 48 horas do pósoperatório com o objetivo de evitar-se hipotensão arterial.

As sessões de hemodiálise foram realizadas três vezes por semana, com fluxo sangüíneo calculado para cada paciente de acordo com a fórmula: $(2,5 \mathrm{x}$ peso $)+100 \mathrm{ml} / \mathrm{min}$.

Foram anotadas as complicações agudas e tardias associadas ao procedimento cirúrgico, assim como analisados os fatores acima citados relacionados às complicações. Dos 14 pacientes que estavam sendo submetidos à hemodiálise, 11 foram entrevistados isoladamente, dois não foram localizados para entrevista e uma paciente morreu devido à falência de múltiplos órgãos após transplante renal.

A equipe de enfermagem do setor de Hemodiálise/ HC/UNICAMP foi entrevistada quanto ao grau de dificuldade para acessar as FAV.

Os resultados foram tabulados e submetidos ao teste do qui-quadrado com nível de significância a = 0,05.

\section{RESULTADOS}

Das 35 FAV confeccionadas (oito proximais e 27 distais), 26 estão pérvias, sendo que 13 pacientes estão em hemodiálise, seis continuam sendo tratados com diálise pe- 
ritoneal, quatro tratados clinicamente e três foram transplantados com sucesso.

A presença de frêmito e/ou pulso logo após o término do procedimento foi observada em $31 \mathrm{FAV}(88,6 \%)$. Ocorreu sucesso imediato em $88,8 \%$ dos pacientes com mais de $20 \mathrm{~kg}$ e de $87,5 \%$ nos pacientes com menos de $20 \mathrm{~kg}$ (Tabela 1). Quatro pacientes evoluíram precocemente sem frêmito local, necessitando confecção de nova FAV, que foi realizada em outra localização, após um período que variou de um a cinco meses.

Tabela 1

Tabela comparativa da evolução das FAV realizadas em crianças com peso inferior ou superior a $20 \mathrm{~kg}$.

\begin{tabular}{lcc}
\hline Peso $(\mathrm{kg})$ & $<20 \mathrm{~kg}$ & $>20 \mathrm{~kg}$ \\
\hline Número de pacientes & 7 & 24 \\
FAV criadas & 8 & 27 \\
FAV proximal & 1 & 7 \\
FAV distal & 7 & 20 \\
Trombose imediata & $1(12,5 \%)$ & $3(11,2 \%)$ \\
Trombose tardia & $1(14,3 \%)$ & $3(12,5 \%)$ \\
Total trombose & $2(25 \%)$ & $6(22,2 \%)$ \\
Patência média (meses) & 13,8 & 14,8
\end{tabular}

Não ocorreu nenhum episódio de trombose entre as oito FAV proximais. Em contrapartida, oito $(29,6 \%)$ das 27 FAV distais foram perdidas às custas de processo trombótico, sendo quatro delas precocemente (menos de um mês). Em uma criança a trombose da FAV ocorreu tardiamente por garroteamento inadvertido do membro, durante procedimento cirúrgico.

Das 23 FAV distais que permaneceram pérvias por mais de três meses, oito estão sendo utilizadas (16,3 meses, em média). Por outro lado, entre as $15 \mathrm{FAV}$ que não foram utilizadas ocorreram quatro tromboses (4/15) $(\mathrm{p}=0,2569$, NS) (Tabela 2). O tempo decorrido entre a confecção e a utilização da FAV variou de duas semanas a 15 meses, dependendo da necessidade clínica do paciente e da disponibilidade de vaga no setor de hemodiálise. A taxa de perviedade da FAV observada a longo prazo em pacientes pesando menos de $20 \mathrm{~kg}$ foi de $75 \%$, sendo que essa mesma taxa foi igual a $77,8 \%$ naqueles pesando mais de $20 \mathrm{~kg}$.

Não foi observado aumento estatisticamente significante no índice de trombose nas FAV confeccionadas em pacientes hipertensos, naqueles com tendência à trombose, hipercolesterolêmicos ou com tendência à hipovolemia quando comparados aos pacientes sem essas patologias.

A boa aceitação da FAV e da hemodiálise foi observada em todos os pacientes entrevistados, assim como a facilidade das punções arteriovenosas pela equipe de enfermagem.
Tabela 2

Patência das FAV distais x utilização.

\begin{tabular}{lcccc}
\hline Patência/Uso & Usa & Não usa & Total \\
\hline Pérvia & 8 & 11 & 19 \\
Trombose tardia & 0 & 4 & 4 \\
\hline Total & 8 & 15 & $23^{*}$ \\
\hline
\end{tabular}

*=Excluindo-se as quatro FAVs distais que trombosaram precocemente.

\section{DISCUSSÃO}

O acesso vascular para hemodiálise deve atender quatro necessidades principais: perviedade de longa duração, respeito às propriedades vasculares, baixas taxas de complicação e boa aceitação pela criança. Todos esses quesitos são bem atendidos pela técnica inicialmente descrita por Brescia e Cimino ${ }^{2}$, e utilizada na confecção das FAV na presente série.

Em 1970 Wander et al. ${ }^{14}$ descreveram os dez primeiros casos de confecção bem-sucedida de FAV em crianças. Ainda, em 1973, apenas 54\% das FAV feitas em crianças com menos de $20 \mathrm{~kg}$ ficavam patentes. Nessa época, Yazbeck ${ }^{11}$ e Bourquelot ${ }^{7}$ chamaram a atenção para a importância de técnicas microcirúrgicas, pois com elas era possível a obtenção de $90-100 \%$ de sucesso, mesmo em pacientes com menos de $10 \mathrm{~kg}$. Os resultados obtidos pelos autores foram considerados aceitáveis quando comparados à literatura pesquisada, demonstrando índices semelhantes de sucesso, tanto precoces quanto tardios (Tabela 3 ).

Com a utilização de técnicas microcirúrgicas, a maioria dos acessos vasculares pôde ser realizada facilmente no antebraço distal da criança, com resultados semelhantes àqueles encontrados em adultos. A técnica microcirúrgica com uso de lupas, boa iluminação, dissecção precisa e uso de sutura com fio 7-0, evita a necessidade de dilatação e o risco de lesão da íntima vascular.

$\mathrm{Na}$ série analisada, a porcentagem de perda da FAV foi de $25 \%$ nos pacientes com menos de $20 \mathrm{~kg}$ e $22,2 \%$ nos pacientes com mais de $20 \mathrm{~kg}$. Em alguns casos, já no intraoperatório pôde-se observar fatores que poderiam levar ao insucesso da FAV, como por exemplo o calibre muito reduzido dos vasos ou vasos de paredes fibróticas.

Todas as FAV utilizadas para sessões de hemodiálise estão pérvias. As FAV que não foram utilizadas e que permaneceram pérvias por mais de três meses, apresentaram $23,5 \%$ de trombose. Isto pode sugerir que a utilização da FAV, produzindo hiperfluxo sangüíneo, turbilhonamento local e dilatação vascular durante a sessão de hemodiálise, favoreça a manutenção da viabilidade da FAV. Por isso, sugere-se que a confecção da FAV se dê numa fase da doença onde a hemodiálise esteja próxima de ser indicada.

Os pacientes que apresentaram perda da FAV no pós-operatório imediato, tinham em comum vasos de calibre muito reduzido, tanto arterial quanto venoso, o que 
Tabela 3

Patência imediata e tardia descrita por diferentes autores (PI=patência imediata; P>3m=Patência maior do que 3 meses)

\begin{tabular}{|c|c|c|c|c|c|c|}
\hline & $F A V<20 \mathrm{~kg}$ & $F A V>20 \mathrm{~kg}$ & $P I<20 \mathrm{~kg}$ & $P I>20 \mathrm{~kg}$ & $P>3 m<20 \mathrm{~kg}$ & $P>3 m>20 k g$ \\
\hline Sicard $^{9}$ & 8 & 25 & $87 \%$ & $80 \%$ & $87,5 \%$ & $64 \%$ \\
\hline Arbus $^{4}$ & 8 & 54 & $50 \%$ & $66 \%$ & $37,5 \%$ & $50 \%$ \\
\hline Gagnadoux ${ }^{10}$ & 66 & 77 & $64 \%$ & $72 \%$ & - & - \\
\hline Kin Neart ${ }^{5}$ & 3 & 10 & $66 \%$ & $90 \%$ & - & - \\
\hline Bourque Lot $^{7}$ & 30 & - & $90 \%$ & - & $62,5 \%$ & - \\
\hline Yazbeck $^{9}$ & 10 & 13 & $100 \%$ & $100 \%$ & $90 \%$ & $100 \%$ \\
\hline Bagolan $^{16}$ & 20 & 70 & $89 \%$ & $90 \%$ & $70 \%$ & $90 \%$ \\
\hline Unicamp & 8 & 27 & $87,5 \%$ & $88,8 \%$ & $71,4 \%$ & $75 \%$ \\
\hline
\end{tabular}

provavelmente propiciou redução no fluxo sangüíneo local, favorecendo processo trombótico.

Embora sem atingir significância estatística, pôde ser constatada, no presente estudo, uma maior tendência à trombose nas FAV distais, o que pode ser explicado pelo menor calibre dos vasos anastomosados ${ }^{15}$.

No presente estudo não houve diferença na perviedade de FAV confeccionadas em pacientes com HAS, antecedentes de trombose, tendência à hipovolemia ou à hipercolesterolemia quando comparados aos pacientes sem estas patologias.

Os grau de satisfação dos pacientes, seja com o aspecto da FAV ou de sua utilização é bastante satisfatório, e todos eles mostraram-se favoráveis à utilização da mesma.

A equipe de enfermagem referiu maiores dificuldades para punção de FAV em crianças em relação à punção em adultos, porém este fato não inviabilizou a utilização da mesma.
Os dados apresentados, com as limitações impostas pelo pequeno número de caso, permitem concluir que:

1) A confecção de FAV é tecnicamente factível em crianças, com resultados comparáveis à literatura pertinente.

2) Todas as fístulas proximais estão pérvias; em contrapartida, ocorreu trombose em 1/3 das fístulas distais, o que pode ser explicado pelo seu menor diâmetro.

3) Parece existir uma melhor evolução das FAVs nos pacientes que a utilizam.

4) Não foi demonstrado maior risco de trombose da FAV em pacientes hipertensos, com antecedente de trombose, tendência à hipovolemia ou à hipercolesterolemia em relação aos pacientes sem essas patologias. O peso dos pacientes também não foi fator determinante na perviedade das FAVs.

\begin{abstract}
Background: Since the development of operative techniques for arteriovenous fistula (AVF) creation, chronic hemodialysis for treatment of end-stage renal disease in children and young adults became an actual therapeutic option, obviating the need for external shunts. Purpose: To report our experience with surgically created AVF in 31 patients, aged 5 to 24 years old, relating long-term fistula patency with risk factors such as arterial hypertension, previous thrombosis events, tendency to hypovolemy, hypercholesterolemy, vein caliber and routine use of the fistula. Results: Overall rate of fistula thrombosis was 22,8\%, being $25 \%$ in patients weighing less than $20 \mathrm{~kg}$ and 22,2\% in those over 20kg. All fistulas performed on proximal arm veins were patent after 5 to 32 months follow-up. Unused AV fistulas apparently had an increased risk of thrombosis when compared to fistulas routinelly used for hemodialysis access. None of the remainy risk factors studied showed estatistical association with loss of patency. Conclusion: AV fistulas can be safely performed in children with success rate comparable to those observed in older patients. Vein caliber and routine use of the fistula appears to have a strong influence on long term patency.
\end{abstract}

Key Words: Arteriovenous fistula; hemodialysis; pediatrics; microsurgery.

\title{
REFERENNCIAS
}

1. Quinton WE, Dillard D, Scribner BH - Cannulation of blood vessels for prolonged hemodialysis. Tr Am Soc Artif Intern Organs 1960; 6:104-113.
2. Brescia MJ, Cimino JE, Appel K, et al - Chronic hemodialysis using venipuncture and a surgically created arteriovenous fistula. $N$ Engl J Med 1966; 275:1089-1092. 
3. Raimbault,G - Psychological aspects of chronic renal failure and hemodialysis. Nephon 1973; 11: 251-260.

4. Hardy MA, Schneider KM, Levitt SB - An improved technique for the construction of internal arteriovenous fistulae in uremic children. J Pediatr Surg 1974; 9: 465-466.

5. Kinnaert P, Janssen F, Hall M - Elbow arteriovenous fistula for chronic hemodialysis in small children. Jour Ped Surg. 1983; 18:116-118.

6. Gracz KC, Ing TS, Soung LS, et al - Proximal forearm fistula for maintenance hemodialysis. Kidney Int. 1977; 11:711-4.

7. Bourquelot P, Wolfeler L, Lamy L - Microsurgery for hemodialysis distal arteriovenous fistulae in children weighing less than 10kg. Proc EDTA 1981; 18:53741.

8. Arbus GS, De Maria JE, Galiwango J, et al - The first 10 years of the dialysis-transplantation program at the Hospital of Sick Children, Toronto. I. Pre-dialysis and dialysis. Can. Med. Ass. J. 1980; 120:655-659.

9. Sicard GA, Merrell RC, Etheredge EE, et al. - Subcutaneous arteriovenous dialysis fistulas in pediatric patients. Trans. Am. Soc. Artif. Internal Organs 1978; 24:695697.

10. Gagnadoux MF, Pascal B, Bronstein M, et al. - Arteriovenous fistulae in small children. Proc. EDTA 1978; 15:573-574.

11. Kinnaert P, Janssen F, Hall N, et al. - Utilisation des fistules astérioveneuses pour l'hémodialyse chez l'enfant. Chir. Pédiatr. 1978; 19:233-237.
12. Yazbeck S, $\mathrm{O}^{\prime}$ Regan S - Microsurgery for Brescia-Cimino Fistula Construction in Pediatric Patients. Nephron 1984; 38:209-212.

13. Wander JV, Moore ES, Jonasson O - Internal arteriovenous fistulae for dialysis in children. J. Pediat. Surg. 1970; 5:533-538.

14. Mauer SM, Lynch RE - Hemodialysis techniques for infants and children. Pediat Clin N Am. 1976; 23:8443-56.

15. Bagolan P, Spagnoli A, Cipriandi G, et al. - A ten-year experience of Brescia-Cimino arteriovenous fistula in children: Technical evolution and refinements. J. Vasc. Surg. 1998; 27:640-644.

Endereço para correspondência:

Dra. Márcia Alessandra Cavalaro

Rua Pedro Vieira da Silva, 64, C- 53

13080-570 - Campinas-SP

E-mail: mcavalaro@yahoo.com 